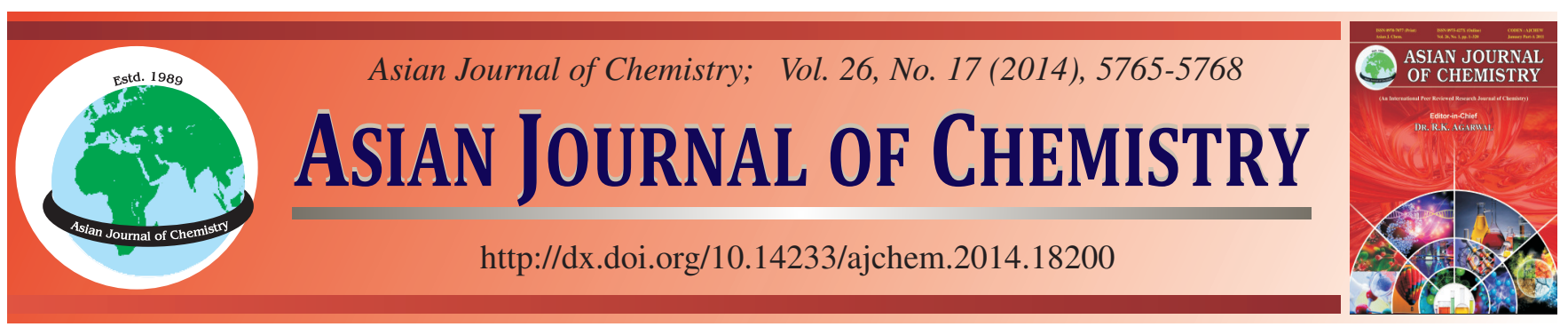

\title{
Pharmacokinetics of Colon-Targeted Pellets of Pulsatilla chinensis (Bunge) Regel Saponins-Hydroxypropyl- $\beta$-cyclodextrin Inclusion in Rats $\dagger$
}

\author{
Zhenhua Chen ${ }^{1, *}$, Yongmei Guan ${ }^{2}$, Ming YAnG ${ }^{2}$, Shilin YAng ${ }^{2}$ and Hongning Liu ${ }^{2}$
}

${ }^{1}$ School of Pharmacy, Jiangxi Science and Technology Normal University, Nanchang, P.R. China

${ }^{2}$ Key Laboratory for Modern preparation of Traditional Chinese Medicine, Ministry of Education, Jiangxi University of Traditional Chinese Medicine, Nanchang, P.R. China

*Corresponding author: Fax: +86 791 83805385; Tel: +86 791 83802393; E-mail: zhenhuadeai@163.com

AJC-15789

\begin{abstract}
The colon-targeted pellets of Pulsatilla chinensis saponins-hydroxypropyl- $\beta$-cyclodextrin inclusion was investigated for the pharmacokinetics study in rats. A rapid and sensitive assay was developed and validated using liquid chromatography-mass spectrometry (LC-MS) for Pulsatilla saponin D in rat plasma. Pulsatilla saponin D was extracted with acetonitrile from rat plasma samples and the supernatants was dried in a gentle stream of nitrogen and redissolved with methanol. It was separated using HPLC with a reversed phase column and analyzed by selected ion monitoring (SIM) at m/z of 935.4 and 969.4 for Ginsenoside Re as an internal standard. The Pulsatilla saponin D concentration was measured in plasma samples up to $24 \mathrm{~h}$ following oral administration of Pulsatilla chinensis saponins and the colon-targeted pellets of Pulsatilla chinensis saponins-hydroxypropyl- $\beta$-cyclodextrin inclusion both at a dose of $200 \mathrm{mg}$ $\mathrm{kg}^{-1}$. The findings indicate that it can decrease the absorption rate and maximum concentration, at the same time improve the bioavailability by preparing colon-targeted pellets of hydroxypropyl- $\beta$-cyclodextrin inclusion complex.
\end{abstract}

Keywords: Pulsatilla chinensis (Bunge) regel saponins, Colon-targeted pellets, LC-MS, Pharmacokinetics, Pulsatilla saponin D.

\section{INTRODUCTION}

Pulsatilla chinensis (Bunge) Regel is a botanical with a long history of medicinal use in China, which exhibits "bloodcooling" and detoxification activities especially in colon and was used in traditional Chinese medicine for the treatment of ulcerative colitis (UC) $)^{1,2}$. Saponins are triterpene or steroid glycosides found in a wide variety of plants as well as in $P$. chinensis ${ }^{3}$. Recent research showed that $P$. chinensis saponins possess an effect of anti ulcerative colitis ${ }^{4}$ and can be developed to a new kind of drug for treatment of ulcerative colitis. However, it should be used through retention-enema to obtain better effect ${ }^{5}$, so that its application was limited attributed to the inconvenience.

The oral colon targeting system refers to the system, in which orally administered medicines are kept from releasing in the upper digestive tract until they are transited to the cecum or colon so that they can exert a local effect on the disease region to improve the therapeutic effect and decrease toxic or adverse action by a convenient administration ${ }^{6}$. Because of the low solubility of $P$. chinensis saponins ${ }^{7}$, its inclusion complex was prepared with a kind of hydrophilic polymer material hydroxypropyl- $\beta$-cyclodextrin and then the colon-targeted pellets were made of the inclusion complex, which were then coated with $\mathrm{pH}$-sensing material polymethyl poly(methyl) acrylates Eudragit S100 and showed a good colon-targeted drug release characteristic in vitro in our previous study ${ }^{8}$.

The objective of the present study was to develop and validate a method to assay hederagenin $3-\mathrm{O}-\alpha-\mathrm{L}-$ rhamnopyranosyl $(1 \rightarrow 2)$ - $[\beta$-D-glucopyranosyl $(1 \rightarrow 4)]$ - $\alpha$-L-arabinopyranoside in rat plasma, which is the main components of $P$. chinensis saponins ${ }^{3}$ and compare the pharmacokinetic characteristic of colon-targeted pellets of $P$. chinensis saponinshydroxypropyl- $\beta$-cyclodextrin inclusion with $P$. chinensis saponins in rats.

\section{EXPERIMENTAL}

The materials used together with the suppliers were as follows: coated pellets of $P$. chinensis (Bunge) Regel saponinshydroxypropyl- $\beta$-cyclodextrin inclusion (PRS-HPCD, selfprepared), standard hederagenin 3-O- $\alpha$-L- rhamnopyranosyl $(1 \rightarrow 2)$-[ $\beta$-D-glucopyranosyl $(1 \rightarrow 4)]-\alpha$-L-arabinopyranoside (Pulsatilla saponin D, purity $>98 \%$, National Pharmaceutical Engineering Center for Manufacturing Solid Preparation of 
Chinese Materia Medica), Ginsenoside Re as an internal standard (purity $>99 \%$, National Institutes for Food and Drug Control), acetonitrile and methanol (HPLC grade, TEDIA company, LNC. USA), formic acid (HPLC grade, SigmaAldrich), first class Sprague-Dawley rats (male and female, weight 180-220 g); 6400 Liquid chromatography-mass spectrometry instrument (Angilent, USA). All solvents were HPLC grade and were used without further purification.

Chromatography and mass spectrometry operating conditions: The separation of Pulsatilla saponin D and the internal standard from endogenous substances was performed on a reversed phase chromatogram column: Hypersil $\mathrm{ODS}_{2}$ $\mathrm{C}_{18}(5 \mu \mathrm{m}, 250 \mathrm{~mm} \times 4.6 \mathrm{~mm})$. The mobile phase consisted of acetonitrile and $0.1 \%$ formic (from 30:70 to 56:44 within 0-6 $\mathrm{min}$ ) at a flow rate of $0.4 \mathrm{~mL} \mathrm{~min}^{-1}$. The injection volume was $0.02 \mathrm{~mL}$ and the analytical column was maintained at $30^{\circ} \mathrm{C}$ during analysis.

Mass spectrometric detection was performed with an ESI source in positive-ion mode. The settings of the mass spectrometer for Pulsatilla saponin D and internal standard were $135 \mathrm{~V}$ for transmission voltage, $10 \mathrm{~L} \mathrm{~min}^{-1}$ for flow rate of drying gas, 40 psi for atomization pressure and $350{ }^{\circ} \mathrm{C}$ for drying gas temperature. In this study, the primary ions for Pulsatilla saponin D and internal standard were monitored simultaneously at $\mathrm{m} / z, 935.4$ and 969.4, respectively.

Sample preparation: $0.1 \mathrm{~mL}$ of rat plasma sample were added with $0.02 \mathrm{~mL}$ internal standard solution $\left(1.0 \mu \mathrm{g} \mathrm{mL}^{-1}\right)$ and $0.3 \mathrm{~mL}$ acetonitrile. The mixture was vortex-mixed for $5 \mathrm{~min}$. The supernatant was obtained after centrifugation for $15 \mathrm{~min}$ at 16,000 rpm and additional supernatant was got out of the residual by the same method as above. The two supernatants were mixed and dried under a stream of nitrogen and re-dissolved with $0.2 \mathrm{~mL}$ methanol, of which $0.02 \mathrm{~mL}$ were injected into the LC-MS system.

Preparation of standards and quality control (QC) samples: Stock solutions of Pulsatilla saponin D and internal standard were prepared in methanol at concentrations of 427.44 and $400.00 \mu \mathrm{g} \mathrm{mL}{ }^{-1}$, respectively. A set of Pulsatilla saponin D solutions and internal standard solutions were obtained by successive dilutions of the stock solutions with methanol. A $0.02 \mathrm{~mL}$ aliquot of Pulsatilla saponin D standard solutions with different concentrations and $0.02 \mathrm{~mL}$ internal standard solutions $\left(1.0 \mu \mathrm{g} \mathrm{mL}^{-1}\right)$ was spiked into $0.1 \mathrm{~mL}$ of blank rat plasma, resulting in seven calibration standards with Pulsatilla saponin D concentrations of 2.14, 10.68, 21.36, 106.8, 213.72, 534.32 and $1068.6 \mathrm{ng} \mathrm{mL}^{-1}$. QC samples with concentrations of Pulsatilla saponin D of 10.68, 106.8 and $1068.6 \mathrm{ng} \mathrm{mL}^{-1}$ were prepared in bland plasma.

\section{Method validation}

Selectivity: The selectivity of the analysis was evaluated using blank plasma, plasma containing Pulsatilla saponin D and internal standard to confirm the absence of endogenous interfering peaks in the chromatograms. The limit of detection was set at the concentration having a signal-to-noise ration of 3 .

Linearity: Calibration curves were constructed for peak area ratio of Pulsatilla saponin D to internal standard versus the concentration of Pulsatilla saponin D in plasma standards.
Concentration ranged from 2.14 to $1068.6 \mathrm{ng} \mathrm{mL}^{-1}$. The calibration curves were fitted by un-weighted linear least-squares regression.

Accuracy and precision: Three batches prepared on different days were used to assess the accuracy and precision of the assay. Analyses of each batch consisted of running calibration and five replicate quality control samples at concentration of $10.68,106.8$ and $1068.6 \mathrm{ng} \mathrm{mL}^{-1}$. The precision was defined as the coefficient of variation at each concentration and the accuracy was determined by calculating the difference between the calculated and theoretical concentrations.

Absolute recovery: Absolute recoveries of Pulsatilla saponin D were determined by comparing the mean peak areas of the analytes added before extraction into the same samples with those of the analytes added to post-extraction samples from different lots of rat plasma at three concentrations.

Stability: The stability of Pulsatilla saponin D in rat plasma after freeze-thaw cycles was evaluated. Quality control samples at concentration of 10.68, 106.8 and $1068.6 \mathrm{ng} \mathrm{mL}^{-1}$ were frozen at $-20{ }^{\circ} \mathrm{C}$ and after 4 days and 7 days thawed at $37^{\circ} \mathrm{C}$, respectively.

\section{Pharmacokinetic study of PRS and colon-targeted pellets of PRS-HPCD in rats}

Administrative method of PRS and PRS-HPCD pellets: The rats in this study were assigned randomly to 2 groups, with six rats in each. The first groups are categorized as the PRS-HPCD colon-targeted pellets experiment groups and the second as the control group. In the control group, PRS was suspended in phosphate buffer ( $\mathrm{pH}$ 5.8) and administered orally by gavage needle, while in the experiment group, PRS-HPCD pellets were administered by home-made gavage instrument directly, both at a dosage of $200 \mathrm{mg} \mathrm{kg}^{-1}$. Heparinized whole blood was sampled from the orbit of rats in the two groups at $0.25,0.5,0.75,1,2,4,6,8,10,12$ and $24 \mathrm{~h}$ after oral administration, respectively. The plasma was separated by centrifugation $(4,000 \mathrm{rpm}, 10 \mathrm{~min})$ and stored at $-20^{\circ} \mathrm{C}$ until analysis.

Statistical analysis: The plasma samples were treated by the process described above and then injected to the LC-MS system. The plasma concentration versus time data was analyzed with one-compartment model using the pharmacokinetics software DAS2.0. The maximum concentration $\left(\mathrm{C}_{\max }\right)$ and the time to reach $\mathrm{C}_{\max }\left(\mathrm{T}_{\max }\right)$ were obtained directly from the profile.

\section{RESULTS AND DISCUSSION}

Specificity, limit of detection and linearity of the calibration curve: Pulsatilla saponin D and internal standard were separated from interfering substances in the plasma under the LC-MS conditions used as shown in Fig. 1. The limit of detection of Pulsatilla saponin D in rat plasma was $0.5 \mathrm{ng}$ $\mathrm{mL}^{-1}$ with a signal-to-noise ratio of 3 . The calibration curve for Pulsatilla saponin D in rat plasma was shown in Fig. 2, which was linear from 2.14-1068.6 $\mathrm{ng} \mathrm{mL}^{-1}$ with a correlation coefficient of $0.9992(y=0.0049 x+0.0058)$.

Accuracy, precision and absolute recovery: As shown in Table-1, within-day precision relative standard deviations (RSD) of plasma sample of high, medium and low concentrations were smaller than $10 \%$, while day-to-day precision RSD smaller than $15 \%$. The accuracy of all the samples was 
TABLE-1

ACCURACY, PRECISION AND ABSOLUTE RECOVERY OF QUALITY CONTROL SAMPLES $(\mathrm{n}=5)$

\begin{tabular}{cccccc}
\hline $\begin{array}{c}\text { Added concentration } \\
\left(\mathrm{ng} \mathrm{mL}^{-1}\right)\end{array}$ & $\begin{array}{c}\text { Accuracy } \\
(\%)\end{array}$ & $\begin{array}{c}\text { Within-day precision } \\
(\%)\end{array}$ & $\begin{array}{c}\text { Day-to-day precision } \\
(\%)\end{array}$ & \multicolumn{2}{c}{ Absolute recovery } \\
\cline { 5 - 6 } & 101.4 & 4.8 & 12.1 & 72.2 & Recovery $(\%)$ \\
10.68 & 97.4 & 7.2 & 9.4 & 71.6 & 9.5 \\
106.8 & 93.1 & 6.3 & 13.5 & 73.1 & 5.1 \\
1068.6 & & &
\end{tabular}
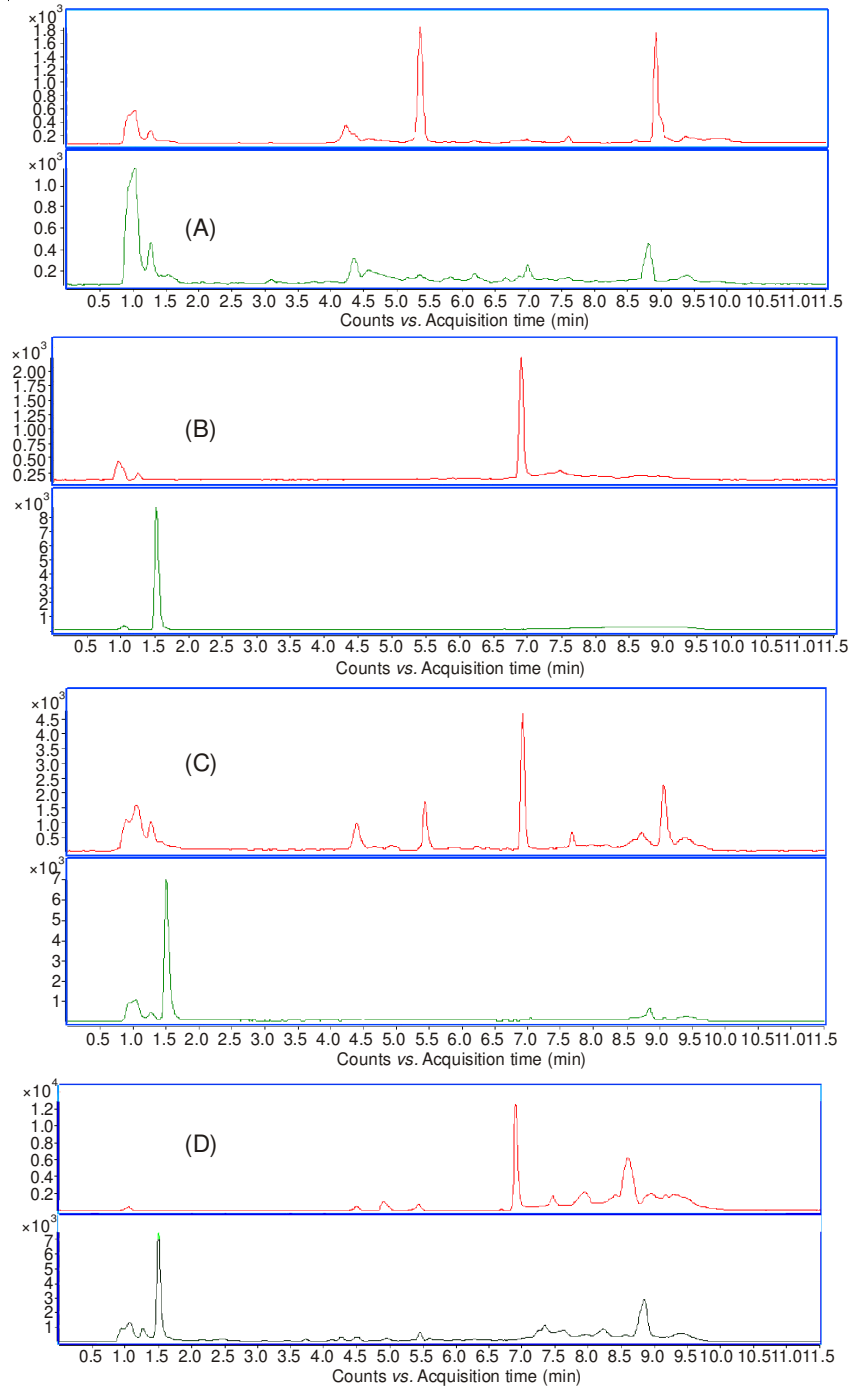

Fig. 1. Chromatograms. (A) blank plasma; (B) mixture of standard Pulsatilla saponin D (upper panel, $\mathrm{t}_{\mathrm{R}}=6.8 \mathrm{~min}$ ) and internal standard (lower panel, $\mathrm{t}_{\mathrm{R}}=1.5 \mathrm{~min}$ ); (C) plasma containing Pulsatilla saponin $\mathrm{D}$ and internal standard; (D) plasma sample obtained from rat after oral administration

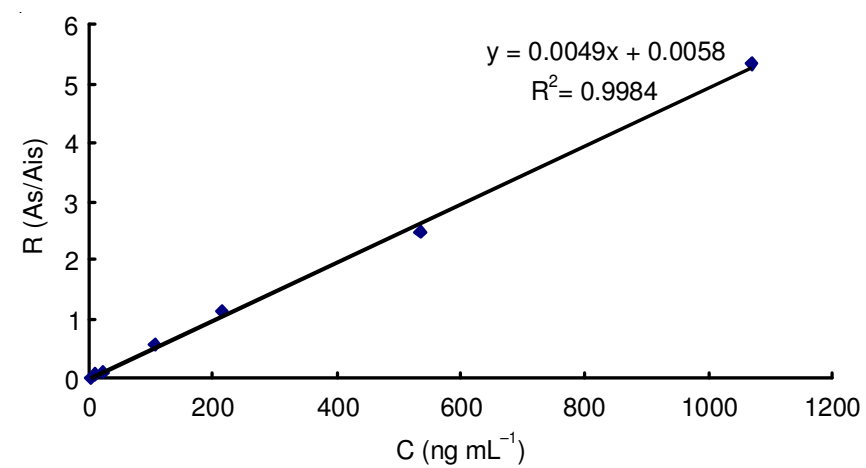

Fig. 2. Calibration curves for Pulsatilla saponin D in rat plasma between 90 and $110 \%$. While the absolute recovery of Pulsatilla saponin D was more than $70 \%$. Collectively, these observations indicate that the current sample processing conditions support adequate demand for the analyte.

Stability: The results showed in Table- 2 indicate that the freeze-thaw cycles after 4 days and 7 days for storage had no effect on the stability of Pulsatilla saponin D as evidenced by the fact that the estimated concentrations differed only slightly from the theoretical values. Therefore, these observations indicate that Pulsatilla saponin D is stable under handling and storage conditions used in the study and that typical processing and storage conditions do not affect the estimation of Pulsatilla saponin D concentration in rat plasma samples.

\begin{tabular}{|c|c|c|c|c|}
\hline \multicolumn{5}{|c|}{$\begin{array}{c}\text { TABLE-2 } \\
\text { STABILITY OF QUALITY CONTROL SAMPLES }(\mathrm{n}=3)\end{array}$} \\
\hline \multirow{2}{*}{$\begin{array}{c}\text { Added } \\
\text { concentration } \\
\left(\mathrm{ng} \mathrm{mL}^{-1}\right)\end{array}$} & \multicolumn{2}{|c|}{$4^{\text {th }}$ day } & \multicolumn{2}{|c|}{$7^{\text {th }}$ day } \\
\hline & $\begin{array}{l}\text { Found } \\
\left(\mathrm{ng} \mathrm{mL}^{-1}\right)\end{array}$ & $\begin{array}{l}\text { RSD } \\
(\%)\end{array}$ & $\begin{array}{l}\text { Found } \\
\left(\mathrm{ng} \mathrm{mL}^{-1}\right)\end{array}$ & $\begin{array}{l}\text { RSD } \\
(\%)\end{array}$ \\
\hline 10.68 & 10.94 & 4.6 & 10.58 & 5.3 \\
\hline 106.8 & 99.76 & 6.2 & 95.22 & 4.7 \\
\hline 1068.6 & 1020.44 & 5.5 & 981.70 & 9.1 \\
\hline
\end{tabular}

Pharmacokinetics of PRS or PRS-HPCD pellets: The assay described above was applied to a pharmacokinetics study of PRS and colon-targeted pellets of PRS-HPCD after oral administration at a dose of $200 \mathrm{mg} \mathrm{kg}^{-1}$ to rats. The mean concentration time profile and the pharmacokinetic parameters are shown in Fig. 3 and Table-3, respectively. The results indicate that after the administration of PRS, Pulsatilla saponin $\mathrm{D}$ was absorbed rapidly to enter the blood circulation. Its blood concentration peak was present at $1 \mathrm{~h}$ after administration and was about $466.13 \mathrm{ng} \mathrm{mL}^{-1}$. By contrast, after the administration of the colon-targeted pellets of PRS-HPCD, the absorption rate was decreased with maximum concentration of 178.35 $\mathrm{ng} \mathrm{mL} \mathrm{m}^{-1}$. The relative bioavailability (BA) of Pulsatilla saponin $\mathrm{D}$ from the colon-targeted pellets of PRS-HPCD is about 3 times of PRS by comparing the AUC, which is opposite to the results in most colon-targeted delivery system, while it may due to the inclusion of hydroxypropyl- $\beta$-cyclodextrin as a hydrophilic material.

\section{Conclusion}

An LC-MS assay for determining Pulsatilla saponin D levels in rat plasma was developed, which took less than $1 \mathrm{~h}$ to detect the blood concentration and validated in the terms of the selectivity, linearity, accuracy, precision, recovery and stability. The pharmacokinetics study of PRS and colontargeted pellets of PRS-HPCD indicate that it can decrease the absorption rate and max concentration by preparing colontargeted pellets of hydroxypropyl- $\beta$-cyclodextrin inclusion complex. 


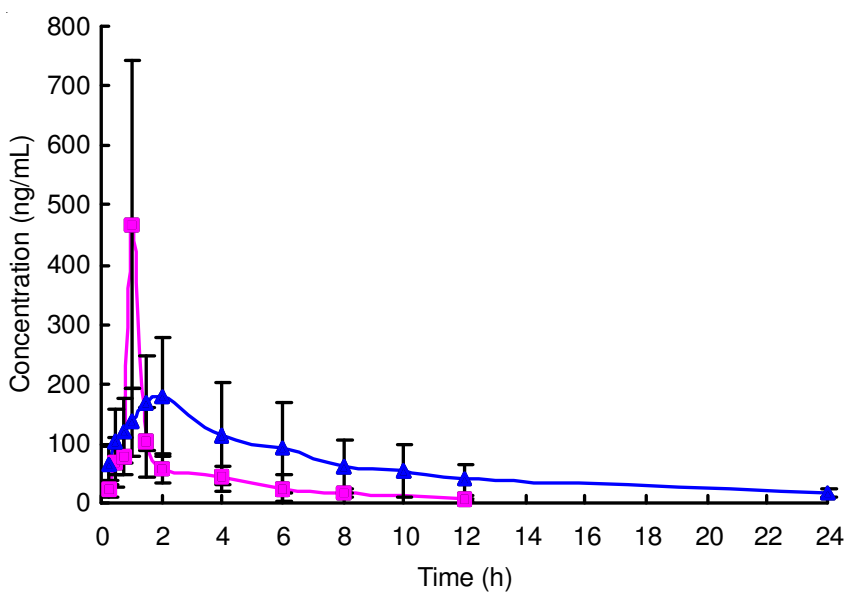

Fig. 3. Mean plasma concentration-time profile of Pulsatilla saponin D following oral administration of PRS ( $\left.\mathbf{\square}, 200 \mathrm{mg} \mathrm{kg}^{-1}\right)$ and colontargeted pellets of PRS-HPCD ( $\boldsymbol{\Lambda}$, equivalent to $200 \mathrm{mg} \mathrm{kg}^{-1} \mathrm{PRS}$ ) to rats. Each point represents the $\overline{\mathrm{x}} \pm \mathrm{s}$ of six experiments.

\section{TABLE-3}

PHARMACOKINETIC PARAMETERS OF Pulsatilla SAPONIN D FOLLOWING ORAL ADMINISTRATION OF PRS AND COLON-TARGETED PELLETS OF PRS-HPCD AT A DOSE OF $200 \mathrm{mg} \mathrm{kg}^{-1}$ IN RATS $(n=6)$

\begin{tabular}{|c|c|c|}
\hline \multirow{2}{*}{$\begin{array}{l}\text { Pharmacokinetic } \\
\text { parameters }\end{array}$} & \multicolumn{2}{|c|}{ Mean \pm SD } \\
\hline & PRS & $\begin{array}{c}\text { Colon-targeted pellets } \\
\text { of PRS-HPCD }\end{array}$ \\
\hline $\mathrm{C}_{\max }\left(\mathrm{ng} \mathrm{mL}^{-1}\right)$ & $466.13 \pm 206.87$ & $190.79 \pm 96.98$ \\
\hline $\mathrm{T}_{\max }(\mathrm{h})$ & $1.00 \pm 0.00$ & $1.92 \pm 0.20$ \\
\hline $\mathrm{t}_{1 / 2}(\mathrm{~h})$ & $2.90 \pm 0.28$ & $6.085 \pm 1.30$ \\
\hline 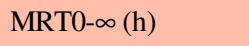 & $3.93 \pm 0.65$ & $13.42 \pm 8.31$ \\
\hline AUC0-t (ng/mL·h) & $542.87 \pm 219.24$ & $1457.20 \pm 906.94$ \\
\hline 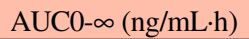 & $574.82 \pm 222.60$ & $1776.32 \pm 1104.37$ \\
\hline
\end{tabular}

\section{ACKNOWLEDGEMENTS}

This work was supported by grant of the Natural Science Foundation of Jiangxi Province (20122BAB215041), the Doctoral Scientific Research Foundation of Jiangxi Science and Technology Normal University (3000990117), the Key Laboratory of Drug Design and Optimization of Jiangxi Science and Technology Normal University (300098010306) and partially by National Scientific and Technology Major Special Project for Significant New Drug Creation (2013ZX09103002-001).

\section{REFERENCES}

1. Z. Wang, N. Zhang and Y. Sun, Drug Eval. Res., 36, 359 (2013)

2. J. Hu, J. Practical Trad. Chinese Internal Med., 22, 29 (2008).

3. W.-C. Ye, N.-N. Ji, S.-X. Zhao, J.-H. Liu, T. Ye, M.A. McKervey and P. Stevenson, Phytochemistry, 42, 799 (1996).

4. W. Zhang, S. Han and H. Yang, Med. J. West China, 17, 99 (2005).

5. Y. Zhuo, Pharmacol. Clinics Chinese Mater. Med., 6, 44 (1990).

6. D.R. Friend, Adv. Drug Deliv., 7, 149 (1991).

7. Z. Chen and Y. Guan, Chinese J. Exper. Trad. Med. Formulae, 18, 28 (2012).

8. Z. Chen, Y. Guan and W. Zhu, China J. Chinese Mater. Med., 38, 4292 (2013). 